\title{
Electrochemical investigation on the film of L-cysteine self-assembled to nanoparticles on a gold electrode
}

\author{
Wenting Wang $^{1}$, Chunming Wang $^{1^{*}}$, Xiaoquan $\mathrm{Lu}^{2^{\star}}$ \\ ${ }^{1}$ Department of Chemistry and Chemical Engineering, Lanzhou University, Lanzhou, China; \\ *Corresponding Author: wangcm@,1zu.edu.cn \\ ${ }^{2}$ Key Laboratory of Bioelectrochemistry \& Environmental Analysis of Gansu Province, College of Chemistry \& Chemical Engineer- \\ ing, Northwest Normal University, Lanzhou, China; ${ }^{*}$ Corresponding Author: luxq@,nwnu.edu.cn
}

Received 27 October 2011; revised 10 December 2011; accepted 21 December 2011

\begin{abstract}
The film contained L-cysteine and gold nanoparticles were provided by self-assembled monolayers (SAMs) and potentiostatic electrodeposition technology on the gold electrode. Two methods were used to study the film: In the first, cyclic voltammetry (CV) was used to inspect the functional groups of the film and the same time hydroquinone was chosen to be a probe molecule in the based solution; secondly, based on analytical technology of scanning electrochemical microscopy (SECM), the heterogeneous rate constant $\left(k_{\text {eff }}\right)$ between solid phase (the modified electrode) and liquid phase $\left(\mathrm{K}_{3} \mathrm{Fe}(\mathrm{CN})_{6}\right)$ was obtained. As a result, the better binary catalysis of hydroquinone was demonstrated and the heterogeneous rate constant $\left(k_{\text {eff }}\right)$ is the greater at 8 $\mathrm{h}$ for L-cysteine self-assembled monolayers (SAMs).
\end{abstract}

Keywords: SECM; L-Cysteine; Hydroquinone; Self-Assembled Monolayers; Heterogeneous Rate Constant

\section{INTRODUCTION}

As well-known, L-cysteine of the favorable electrochemical activity is a common amino acid in the living creature. Because of the special functional end groups, such as $-\mathrm{SH},-\mathrm{COOH}$ and $-\mathrm{NH}_{2}$, it attracted many researchers in different fields deeply. Nano-material has been a hot topic recently. The specific surface area of the modified electrode with nanoparticles by electrochemical technology is larger than that of bare electrode [1]. The new structure of L-cysteine on the Au (111) surface was investigated by situ electrochemical scanning tunneling microscopy [2,3]. Surface-enhanced Raman spectroscopy (SERS) and surface-enhanced second-harmonic generation (SESHG) were employed to investigate the adsorption of L-cysteine on a polycrystalline silver electrode [4]. What matters most was that self-assemble monolayers (SAMs) was a simple and stable method in the study of modified electrode, and it can obtain spontaneously high-order monolayer on gold via the thiol end group [5-8]. The electrode modified directly with Lcysteine SAMs can investigate various performances, including the following several aspects, voltammetric behavior of vitamin $B_{2}$, selective response of dopamine in ascorbic acid and electrochemical behavior of epinephrine [9-11]. Previously, the modified electrode with the biochemical molecular L-cysteine was usually used to catalyze the quinones in the water solution. However, the research on the electrode modified with nanoparticles and L-cysteine is seldom advanced. Moreover, hydroquinone, as electron carrier in photosynthesis and respiration, plays a key role in biological energy metabolism. The electrochemical process of quinone is usually investtigated by cyclic voltammetry (CV) at glassy carbon electrode [12,13], and electroactive quinone as terminal group is immobilized on electrode to investigate the well-know electron transport (ET) [14,15]. As an electrochemical technology, scanning electrochemical microscopy (SECM) is a crucial tool to study electron transfer (ET) at solid/liquid interface, liquid/liquid interface, and it has several advantages over cyclic voltammetry $(\mathrm{CV})$, such as allowing more informations to be extracted from a single measurement [16-18].

Here, we first modified the gold electrode with the gold nanoparticles by potentiostatic electrodeposition and then applied self-assembly method to modify Lcysteine on the gold nanoparticles. SECM was employed to observe heterogeneous phase ET reaction between the electrode and mediator. In the presence of hydroquinone as a probe molecule, $\mathrm{CV}$ was used to investigate electrochemical response of the film, which consisted of gold 
nanoparticles and L-cysteine on the gold electrode,

\section{MATERIAL AND METHODS}

\subsection{Chemicals}

L-cysteine was received from the Factory of Caoyang second middle school (Shanghai, China). Hydroquinoneand $\mathrm{HAuCl}_{4} \cdot 4 \mathrm{H}_{2} \mathrm{O}$ was from Fucheng Chemical Regent Factory (Tianjin, China), Sinopharm Chemical Regent Co., Ltd (Shanghai, China), respectively. All other reagents were analytical-reagent grade, unless otherwise specified. Solutions were prepared from water that had been purified through an Ultra-pure water system MilliQ Plus (Millipore).

\subsection{Preparation of Substrate and SAMs}

A gold electrode (Diameter $=2 \mathrm{~mm}$ ) was used as the substrate for these experiments. The electrode was polished to a mirror finish using $0.05 \mu \mathrm{m}$ alumina powder before careful rinsing with deionized (DI) water, sonication in absolute ethanol and DI water for $10 \mathrm{~min}$ in turn, and then dried with nitrogen gas. The gold electrode was modified with gold nanoparticles by potentiostatic electrodeposition at $-0.2 \mathrm{~V}$ in $3 \mathrm{mM} \mathrm{HAuCl} 4$ solution [1]. SAMs was prepared with a solution of $1 \mathrm{mM} \mathrm{L}$-cysteine in phosphate-buffered solution $(\mathrm{pH}=7)$.

\subsection{Electrochemical Measurements}

Electrochemical experiments were carried out using a CHI 900 scanning electrochemical microscope (CH Instruments Co. Ltd, Austin, USA) with a four-electrode cell. The gold (or modified gold) electrode, a platinum wire, and a $\mathrm{KCl}$ saturated $\mathrm{Ag} / \mathrm{AgCl}$ electrode were used as working, counter and reference electrode, respectively. The SECM tip was a $25-\mu \mathrm{m}$ diameter Pt ultramicroelectrode (UME). Before each experiment, the tip was polished with $0.3-\mu \mathrm{m}$ alumina and rinsed with DI water.

\section{RESULTS}

\subsection{Electrochemical Characterization of the SAMs}

Electrochemical means of cyclic voltammetry (CV) have been performed to characterize the SAMs. Figure 1(A) showed CVs for $1 \mathrm{mM} \mathrm{K}_{3} \mathrm{Fe}(\mathrm{CN})_{6}$ containing 0.1 $\mathrm{M} \mathrm{KCl}$ at a) a clean gold electrode, b) a gold electrode modified with nano gold, c) L-cysteine self-assembled to the nanogold electrode.

For the bare $\mathrm{Au}$ electrode, the typical $\mathrm{CV}$ response shape of ferricyanide was performed. The peak separation of $70 \mathrm{mV}$ showed a reversible voltammogram for the redox couple, indicating a diffusion-limited or electrochemical quasi-reversible one electron redox process.

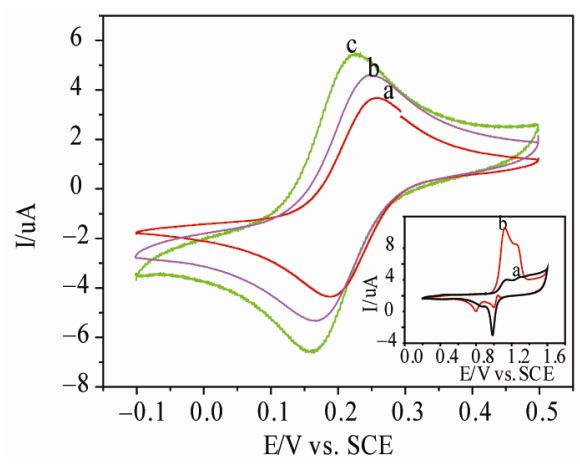

(A)

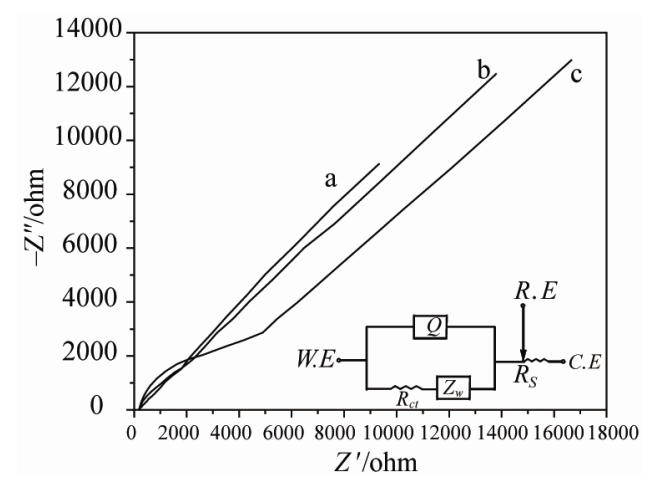

(B)

Figure 1. (A) Cyclic voltammograms in $1 \mathrm{mM}$ $\mathrm{K}_{3} \mathrm{Fe}(\mathrm{CN})_{6}$ at scan rate $100 \mathrm{mV} / \mathrm{s}$. Insert: Cyclic voltammograms in $1 \mathrm{M} \mathrm{H}_{2} \mathrm{SO}_{4}$ at scan rate 50 $\mathrm{mV} / \mathrm{s}$. (B) Impedance spectra corresponding in 1 $\mathrm{mM} \mathrm{K}_{3} \mathrm{Fe}(\mathrm{CN})_{6}$ with $0.1 \mathrm{M} \mathrm{KCl}$ as supporting electrolyte. Insert: The equivalent circuit model used to obtain equations for $Z_{r e}$ and $Z_{i m}$. W.E is the working electrode, R.E is the reference electrode, and C.E is the counter electrode.

When the bare gold electrode was modified with gold nanoparticles, the peak current increased obviously in the solution of either $1 \mathrm{mM} \mathrm{K}{ }_{3} \mathrm{Fe}(\mathrm{CN})_{6}$ or $1 \mathrm{M} \mathrm{H}_{2} \mathrm{SO}_{4}$. Compared with the bare and nanogold electrode, the electrode modified with gold nanoparticles and L-cysteine showed the better reversible $\mathrm{CV}$ response in $\mathrm{K}_{3} \mathrm{Fe}(\mathrm{CN})_{6}$. This may be the protonation of $-\mathrm{NH}_{2}$ in L-cysteine and the electrostatic interaction with $\mathrm{K}_{3} \mathrm{Fe}(\mathrm{CN})_{6}$ [19]. Figure 1(B) showed the electrochemical impedance spectroscopy (EIS) of Nyquist plots. Among them, a) represented the EIS curve of the bare gold electrode, where had a straight line at low-frequency and a small semicircle at a highfrequency region. The phenomenon demonstrated that the process was essentially diffusion-controlled for the redox couple. The electrode modified with gold nanoparticles was used as the working electrode in b). Different from the above dates, c) indicated that L-cysteine had been self-assembled on gold electrode modified with gold nanoparticles previously. 


\subsection{Heterogeneous Rate Constant $\left(k_{\text {eff }}\right)$ by Scanning Electrochemical Microscopy (SECM)}

Figure 2 shows the schematic diagram of the electrochemical cell and the SECM tip.

The $25 \mu \mathrm{m}$ diameter Pt ultramicroelectrode (UME), which is made by our own laboratory, were used to investigate the modified gold electrode surface and record the feedback approach curve at the different concentration of the mediator solution $\left(\mathrm{K}_{3} \mathrm{Fe}(\mathrm{CN})_{6}\right)$. In the SECM experiments, the modified electrode was used as the substrate. Because the terminal groups contain - $\mathrm{COOH}$ and $-\mathrm{NH}_{2}$, we modified electrode with $\mathrm{COOH}$-terminated alkanethiol for the distinction. It was found that the feedback approach curve of SAM with $\mathrm{COOH}$-terminated alkanethiol was negative from $0.5 \mathrm{~V}$ to $0.7 \mathrm{~V}$ (the substrate potential $\left.\left(E_{s}\right)\right)$ vs $\mathrm{Ag} / \mathrm{AgCl}$ with $1 \mathrm{mM} \mathrm{Fe}(\mathrm{CN})_{6}^{3-}$ in $0.1 \mathrm{M} \mathrm{KCl},-0.1 \mathrm{~V}$ (UME tip potential) vs $\mathrm{Ag} / \mathrm{AgCl}$ in Figure 2 (Inser), which was consistent with the previous report [4]. However, the feedback curve for L-cysteine SAMs was just positive at $0.5 \mathrm{~V}$ (the substrate potential $\left.\left(E_{s}\right)\right)$ vs $\mathrm{Ag} / \mathrm{AgCl}$ with $1 \mathrm{mM} \mathrm{Fe}(\mathrm{CN})_{6}^{3-}$ in $0.1 \mathrm{M} \mathrm{KCl}$, $-0.1 \mathrm{~V}$ (UME tip potential) vs $\mathrm{Ag} / \mathrm{AgCl}$, which was attribute to the terminal group: $-\mathrm{NH}_{2}$ in the L-cysteine SAMs on the modified electrode with the nano gold. For this reason, we conjectured that $-\mathrm{NH}_{2}$ in the L-cysteine $\mathrm{SAM}$ was the key role in the ET process between the modified electrode and the mediator $\left(\mathrm{K}_{3} \mathrm{Fe}(\mathrm{CN})_{6}\right)$. Table 1 showed the heterogeneous rate constant $\left(k_{\text {eff }}\right)$ of the modified gold electrode with the different assembling time by L-cysteine.

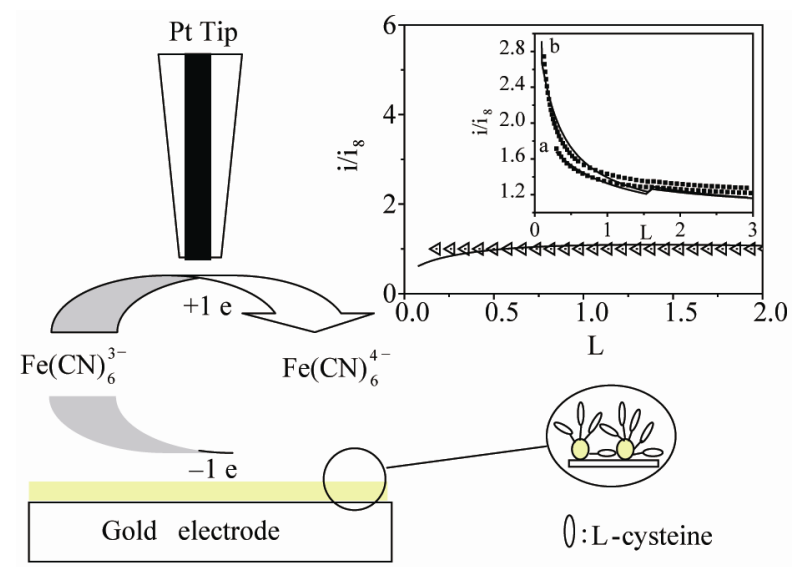

Figure 2. Scheme of the modified electrode and the setup by SECM. Insert: experimental approach curves.

Table 1. Heterogeneous rate constant $\left(k_{\text {eff }}\right)$ of SAMs with Lcysteine on modified electrode with gold nanoparticles.

\begin{tabular}{cccccccc}
\hline Assembling time $(\mathrm{h})$ & 2 & 3 & 4 & 5 & 6 & 7 & 8 \\
\hline$k_{\text {eff }}\left(10^{-3}\right) \mathrm{cm} / \mathrm{s}$ & 6.0 & 7.0 & 6.4 & 6.8 & 6.6 & 6.6 & 7.1 \\
\hline
\end{tabular}

The heterogeneous rate constant $\left(k_{\text {eff }}\right)$ was the greater at $3 \mathrm{~h}$ and $8 \mathrm{~h}$. During the initial stage of self-assembling, the -SH of L-cysteine by Au-S bond attached to the gold nanoparticles in major. The coverage of L-cysteine on gold nanoparticles was relatively large at $3 \mathrm{~h}$ and $\mathrm{Au}-\mathrm{S}$ bond was the main factor. Subsequently, due to the connection between $-\mathrm{NH}_{2}$ and the gold nanoparticles, the Au$\mathrm{NH}$ bond may replace the Au-S bond in a certain degree. The film, including the gold nanoparticles and L-cysteine, reached the new equilibrium at $8 \mathrm{~h}$ and performed the greater heterogeneous rate constant $\left(k_{\text {eff }}\right)$ once again.

\subsection{Cyclic Voltammetry (CV) with Hydroquinone as a Probe Molecule}

Compared to the previous work, the research on the self-assembled time was rarely mentioned. Here, at the same modified condition, we paid the more attention on the assembling time of SAMs.

Figure 3 showed the cyclic voltammograms of hydroquinone modified electrode at different assembling time in acetate buffer solution $(\mathrm{pH}=3.8)$. Obviously, in the first stage $(\mathrm{t}=2 \mathrm{~h}, 3 \mathrm{~h}, 4 \mathrm{~h})$, the oxidation peak had a negative shift and peak current increased at $3 \mathrm{~h}$ (Figure 3(A)). In the next stage ( $\mathrm{t}=5 \mathrm{~h}, 6 \mathrm{~h}, 7 \mathrm{~h}, 8 \mathrm{~h})$, we found that the better change at $8 \mathrm{~h}$ and the oxidation peak had a negative shift and peak current evidently increased (Figure $3(\mathbf{B})$ ). In the further research, we have chosen the three different assembling times, 3 h, 5 h, 8 h (Figure 3 (C)). It showed the oxidation peak position of $3 \mathrm{~h}$ and $8 \mathrm{~h}$ were approximately same. However, the potential difference of $8 \mathrm{~h}$ was the smaller than that of $3 \mathrm{~h}$. Figure 4 showed the three possible SAMs and the reaction with the hydroquinone, where L-cysteine was self-assembled in the phosphate buffer solution $(\mathrm{pH}=6.8)$.

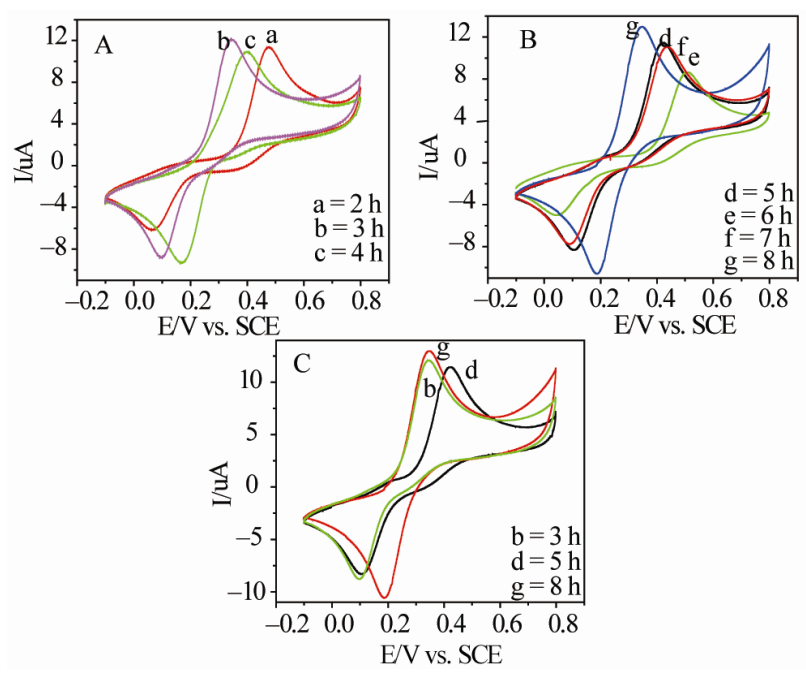

Figure 3. Cyclic voltammograms of the modified gold electrode at different assembling time. 

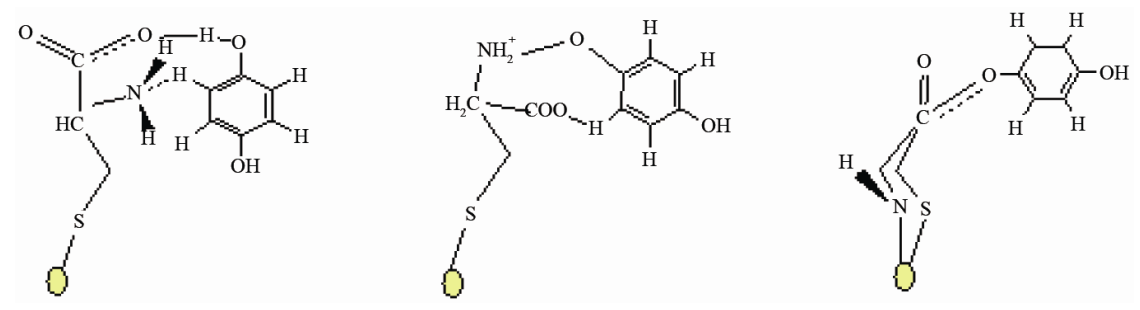

Figure 4. Three different possible SAM and the reaction of hydroquinone.

Because of the existence of the $-\mathrm{COOH}$ and $-\mathrm{NH}_{2}$, the close to neutral solution was chosen for avoiding the effect of $\mathrm{pH}$. Moreover, we supposed that double SAMs of L-cysteine may be formed at the appropriate $\mathrm{pH}$ conditions, namely close to the neutral solution. As above discussion, there were three kinds of the terminal groups in SAMs of L-cysteine: $-\mathrm{NH}_{2},-\mathrm{COOH}$ and $-\mathrm{SH}$, and in the assembling process, the three situations didn't present separately. The CVs of modified electrode in hydroquinone as probe molecule accorded with the heterogeneous rate constant $\left(k_{\text {eff }}\right)$ between the film and the mediator solution. That strongly suggestted quinone could be applied as the probe molecule, and further to learn the film containing some special functional groups on the electrode.

\subsection{Binary Catalysis of the Modified Electrode}

Figure 5 showed the cyclic voltammograms of hydroquinone in acetate buffer solution $(\mathrm{pH}=3.8)$ at the different modification on gold electrode. It was worth to point out that the gold electrode, which was modified with gold nanoparticles and then self-assembled with Lcysteine, performed the preferable capability of catalysis for hydroquinone. From a view point of catalysis, this catalytic reaction should be called binary catalysis, that meant both nano gold and L-cysteine participated the binary catalysis reaction.

\section{CONCLUSION}

In conclusion, based on careful electrochemical analy-

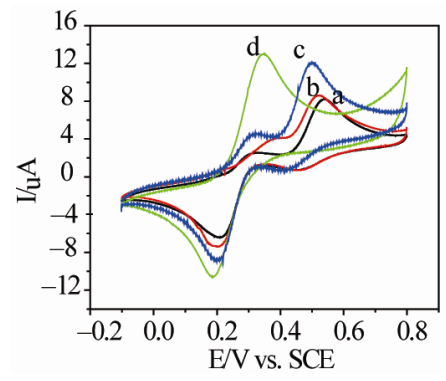

Figure 5. Cyclic voltammograms of gold electrode with different conditions in hydroquinone in acetate buffer solution $(\mathrm{pH}=3.8)$. sis of CVs, it was found that L-cysteine performed the better electrochemical response at $8 \mathrm{~h}$ (self-assembling time) with hydroquinone as a probe molecule in acetate buffer solution $(\mathrm{pH}=3.8)$. The heterogeneous rate constant $\left(k_{\text {eff }}\right)$ obtained from the feedback approach curves of SECM was aslo greater at $8 \mathrm{~h}$. In the low $\mathrm{pH}$-value solution, the functional group which reacted with hydroquinone was mostly $-\mathrm{NH}_{2}$, and $-\mathrm{COOH}$ by the free hydrogen ion. We confirmed that what was the optimal assembling time, and which functional group mainly participated in the reaction. Moreover, the modified electrode with the film performed the binary catalysis in acetate buffer solution of hydroquinone.

\section{ACKNOWLEDGEMENTS}

This work was supported by the Natural Science Foundation of China (No. 20775060, 20875077), the Key Project of Scientific Research Base of education department (08zx-07) and the Key Laboratory of Ploymer Materials of Gansu Province.

\section{REFERENCES}

[1] Liu, S.F., Li, X.H., Li, Y.C., Li, Y.F., Li, J.R. and Jiang, L. (2005) The influence of gold nanoparticle modified electrode on the structure of mercaptopropionic acid self-assembly monolayer. Electrochimica Acta, 51, 427431. doi:10.1016/j.electacta.2005.04.038

[2] Xu, Q.M., Wan, L.J., Wang, C., Bai, C.L., Wang, Z.Y. and Nozawa, T. (2001) New structure of 1-cysteine selfassembled monolayer on $\mathrm{Au}(111)$ : Studies by in situ scanning tunneling microscopy. Langmuir, 17, 6203-6206. doi:10.1021/la010670y

[3] Dakkouri, A.S., Kolb, D.M., Edelstein-Shima, R. and Mandler, D. (1996) Scanning tunneling microscopy study of 1-cysteine on $\mathrm{Au}(111)$. Langmuir, 12, 2849-2852. doi:10.1021/la9510792

[4] Brolo, A.G., Germain, P. and Hager, G. (2002) Investigation of the adsorption of 1-cysteine on a polycrystalline silver electrode by surface-enhanced raman scattering (SERS) and surface-enhanced second harmonic generation (SESHG). Journal of Physical Chemistry B, 106, 5982-5987. doi:10.1021/jp025650z

[5] Lu, X.Q., Zhang, L.M., Li, M.R., Wang, X.Q., Zhang, Y., Liu, X.H. and Zuo, G.F. (2006) Electrochemical characterization of self-assembled thiol-porphyrin monolayers 
on gold electrodes by SECM. ChemPhysChem, 7, 854-862. doi: $10.1002 / \mathrm{cphc} .200500492$

[6] Zhang, S. and Echegoyen, L. (2005) Selective response of dopamine in the presence of ascorbic acid on 1-cysteine self-assembled gold electrode. Journal of the American Chemical Society, 127, 2006-2011. doi:10.1021/ja044411h

[7] Holt, K.B. (2006) Using scanning electrochemical microscopy (SECM) to measure the electron-transfer kinetics of cytochrome c Immobilized on a $\mathrm{COOH}$-terminated alkanethiol monolayer on a gold electrode. Langmuir, 22, 4298-4304. doi:10.1021/la0529916

[8] Wang, W.T., Li, X.J., Wang, X.Y., Shang, H., Liu, X.H. and Lu, X.Q. (2010) Comparative electrochemical behaviors of a series of SH-terminated-functionalized porphyrins assembled on a gold electrode by scanning electrochemical microscopy (SECM). Journal of Physical Chemistry B, 114, 10436-10441. doi:10.1021/jp1026064

[9] Wan, Q.J. Yang, N.J., Zhang, H.L., Zou, X.P. and Xu, B. (2001) Voltammetric behavior of vitamin B2 on the gold electrode modified with a self-assembled monolayer of L-cysteine and its application for the determination of vitamin B2 using linear sweep stripping voltammetry. Talanta, 55, 459-467. doi:10.1016/S0039-9140(01)00437-4

[10] Hu, G.Z., Liu, Y.C., Zhao, J., Cui, S.Q., Yang, Z.S. and Zhang, Y.Z. (2006) Selective response of dopamine in the presence of ascorbic acid on 1-cysteine self-assembled gold electrode. Bioelectrochem, 69, 254-257. doi:10.1016/j.bioelechem.2006.03.005

[11] Wang, S.F., Du, D. and Zou, Q.C. (2002) Electrochemical behavior of epinephrine at L-cysteine self-assembled monolayers modified gold electrode. Talanta, 57, 687-692. doi:10.1016/S0039-9140(02)00072-3

[12] Ji, X.B., Banks, C.E., Silvester, D.S., Wain, A.J. and Compton, R.G. (2007) Electrode kinetic studies of the hydroquinone-benzoquinone system and the reaction between hydroquinone and ammonia in propylene carbonate:
Application to the indirect electroanalytical sensing of ammonia. Journal of Physical Chemistry C, 111, 14961504. doi:10.1021/jp066704y

[13] Quan, M., Sanchez, D., Wasylkiw, M.F. and Smith, D.K. (2007) Voltammetry of quinones in unbuffered aqueous solution: Reassessing the roles of proton transfer and hydrogen bonding in the aqueous electrochemistry of quinones. Journal of the American Chemical Society, 129. 12847-12856. doi:10.1021/ja0743083

[14] Tamaki, T., Ito, T. and Yamaguchi, T. (2007) Immobilization of hydroquinone through a spacer to polymer grafted on carbon black for a high-surface-area biofuel cell electrode. Journal of Physical Chemistry B, 111, 1031210319.doi:10.1021/jp074334n

[15] Larsen, A.G. and Gothelf, K.V. (2005) Electrochemical properties of mixed self-assembled monolayers on gold electrodes containing mercaptooctylhydroquinone and alkylthiols. Langmuir, 21, 1015-1021. doi:10.1021/la048221w

[16] Liu, B., Bard, A.J., Mirkin, M.V. and Creager, S.E. (2004) Electron transfer at self-assembled monolayers measured by scanning electrochemical microscopy. Journal of the American Chemical Society, 126, 1485-1492. doi:10.1021/ja038611p

[17] Lu, X.Q., Nan, M.N., Zhang, H.R., Liu, X.H., Yuan, H.Q. and Yang, J.D. (2007) Investigation of the antioxidant property of ascorbic acid. Journal of Physical Chemistry C, 111, 14998-15002. doi:10.1021/ip072551i

[18] Bollo, S., Yanez, C., Sturm, J., Nunez-Vergara, L. and Squella, J.A. (2003) Cyclic voltammetric and scanning electrochemical microscopic study of thiolated $\beta$-cyclodextrin adsorbed on a gold electrode. Langmuir, 19, 3365-3370. doi:10.1021/la0267995

[19] Hu, X.Y., Xiao, Y. and Chen, H.Y. (1999) Adsorption characteristics of $\mathrm{Fe}_{3}(\mathrm{CN})_{6}{ }^{3-/ 4-}$ on $\mathrm{Au}$ colloids as monolayer films on cysteamine-modified gold electrode. Journal of Electroanalytical Chemistry, 466, 26-30. doi:10.1016/S0022-0728(99)00113-8 